\title{
A Phenomenon of Tenodesis in the Aspect of Gliding Function of Fingers Flexors Tendons
}

\author{
Pieniazek Marek ${ }^{1}$, Krezalek Piotr ${ }^{2}$, Szczechowicz Jakub ${ }^{1 *}$, Jamka Krzysztof ${ }^{4}$, Markowski Andrzej ${ }^{2}$ and Rog \\ Tomasz ${ }^{3}$ \\ ${ }^{1}$ Department of Kinesitherapy, Faculty of Motor Rehabilitation, University of Physical Education in Cracow, Poland \\ ${ }^{2}$ Department of Physical Medicine and Biological Regeneration, Faculty of Motor Rehabilitation, University of Physical Education in Cracow, Poland \\ ${ }^{3}$ Department of Anatomy, Faculty of Motor Rehabilitation, University of Physical Education in Cracow, Poland \\ ${ }^{4}$ The Specialist Centre of the Hand Rehabilitation, Poland
}

Submission: August 05, 2019; Published: August 19, 2019

*Corresponding author: Szczechowicz Jakub, Department of Kinesitherapy, Faculty of Motor Rehabilitation, University of Physical Education in Cracow, Poland

\begin{abstract}
Purpose: Is the motion range in fingers tendons during tenodesis phenomenon dependant on the wrist motion range? Is the tenodesis phenomenon determined by the wrist motion arc?
\end{abstract}

Methods: A three-dimensional analysis of motion was used to determine connections between active movements in the wrist and a range of passive movements induced in such a way within fingers II-V as well as assessing 'efficiency' of tenodesis exercises. The research included a group of 25 students around of age 21 . All participants had to be right-handed and had no former injuries or surgeries within the right hand.

Results: The obtained results demonstrated changes occurring in fingers joints during performed movements in the wrist. The tests showed that in fingers II-V the highest TAM was observed during the wrist movements in the full arc and the lowest in the posterior one. An analysis of the conducted research also indicated presence of differentiated 'efficiency' of tenodesis in individual fingers illustrated by the value of TAM during the wrist movements in the observed arcs.

Conclusion: Differentiated efficiency of the tenodesis phenomenon has been observed in different arcs of the wrist movements. No statistically significant correlation has been found between the indices of TAM describing angular changes occurring in joints of fingers II-V and the range of the wrist movement. Strong correlations of the TAM index have been observed between individual fingers. No significant dependency of the tenodesis phenomenon efficiency from anthropometric indices and muscles force has been demonstrated.

\section{Introduction}

Early application of movement into a rehabilitation program after primary and secondary reconstruction of tendons is an important factor in restitution of functions of individual fingers and the whole hand [1-3]. During the early period after an operation when a risk of complications (including breaking the structures anastomosis) is high, it is suggested that tenodesis exercises should be introduced. They release tendons glide without any necessity of their active load. The phenomenon of tenodesis in relation to fingers flexors tendons relies on active movements of the wrist in the sagittal plane that result in both passive movements in joints and a passive glide of tendons within the fingers. The motions proceed contrary to the wrist movements [4]. The application of glide movements - without an active increase of muscles tensions and loads - in case of tendons anastomosis, qualifies tenodesis exercises to exercises featuring a positive influence on a process of the hand functions restitution. Tenodesis exercises have been applied in about $14.5 \% 8$ of treatment programs in the early phase after an operation. Despite their growing popularity, an optimal standard of performing the exercises has not been elaborated yet, and many authors are doing research aiming at optimization of this form of therapy $[5,6]$.

Maintaining a proper gliding function of fingers flexors tendons is an essential factor of the hand functions. Benefits of an early repair as well as earlier implemented mobilisation have been known for a long time [1-3,7-10]. Differences can be observed in rehabilitation protocols suggested for patients. The ones focusing mainly on passive exercises are being replaced by protocols offering application of active exercises in early phases after tendons reconstruction [11-13]. Improvements in operation techniques and modern suture materials allow to decrease the risk of tendons dehiscence at the anastomosis site. 
It enables application of active movements in earlier and earlier phases of post-surgical rehabilitation. It results in lowering the complications risks like limiting range of fingers motion (articular contracture) or breaking the anastomosis, among others. Besides the proposals, the therapy practice includes also procedures following protocols focused on passive exercises $[8,14,15]$.

The conducted research tests aimed at determining a connection between active movements in the wrist and a range of passive movements induced by this method within fingers II-V as well as assessment of tenodesis exercises effectiveness. Such systemization of knowledge and biomechanical aspects concerning performing tenodesis exercises will allow more effective use of the tenodesis phenomenon in the hand therapy practice and an increase in their proper execution.

\section{Research Questions}

i. Is the motion range in fingers tendons during tenodesis phenomenon dependant on the wrist motion range?

ii. Is the tenodesis phenomenon determined by the wrist motion arc?

\section{Methods}

The tests included 25 students of the University of Physical Education in Cracow. The research group consisted of 17 women and 8 men. The mean age of the examined was 21.2 years (SD 1.02). The following criteria of participation in the tests were defined: dextrality, no former injuries within the right hand, no former surgeries within the right hand.

The research methodology contained a three-dimensional analysis of motion. Recording of the wrist extension and flexion movements and effectiveness of the tenodesis phenomenon assessed based on motion in fingers II - V joints was conducted with the use of a three-dimensional motion analysis BTS-SMART (BTS-Italy). The system contained 6 infrared recording cameras registering waves within the infrared radiation reflected from passive markers placed directly on the examined people's skin. The recording was performed with the frequency of $70 \mathrm{~Hz}$. A set of 26 spherical markers of $5 \mathrm{~mm}$ diameter got placed in anatomical sites on the hand according to the protocol suggested by Metcalf et al. [16]. Repeated extension and flexion movements in the radiocarpal joint with simultaneous extension and flexion movements of fingers were recorded.

The tests were conducted in a sitting position, with the elbow rested against the surface of the lab stand table and the forearm placed vertically. Application of the kinematic model allowed to calculate angles in interphalangeal and metacarpophalangeal joints accompanying angular changes of the wrist. In order to determine total angular changes in fingers II-V during flexion and extension movements, the TAM (Total Active Motion)14 method was applied. The maximal flexion in MCP, PIP and DIP joints was assessed during extension motion of the wrist and extension in the joints was defined during flexion of the wrist. Next, TAM was calculated as a difference between the sum of motion range of fingers II-V flexion in MCP, PIP and DIP joints and extension losses in the joints. The values of TAM were calculated according to the formula:

TAM $=($ range of flexion motion MCP + PIP + DIP $)-($ extension loss MCP + PIP + DIP).

Goniometric measurement of the active motion range of radiocarpal joint was made in order to assess the proper function. Motion ranges of extension and flexion of radiocarpal joint did not present any motion losses in the examined and were in compliance with the norms established by AAOS (American Association of Orthopaedic Surgeons) concerning goniometric measurements of joint motions by SFTR21 method. The obtained results were analysed statistically with the use of the Wolfram Mathematica 9.0 software pack. A descriptive characteristic of the examined group was prepared and matrix correlation as well as difference significance of the obtained results were calculated with the use of the t-Student test for paired groups, defining the statistical significance coefficient at $\mathrm{p}<0.05$ (Figures $1 \& 2$ ).

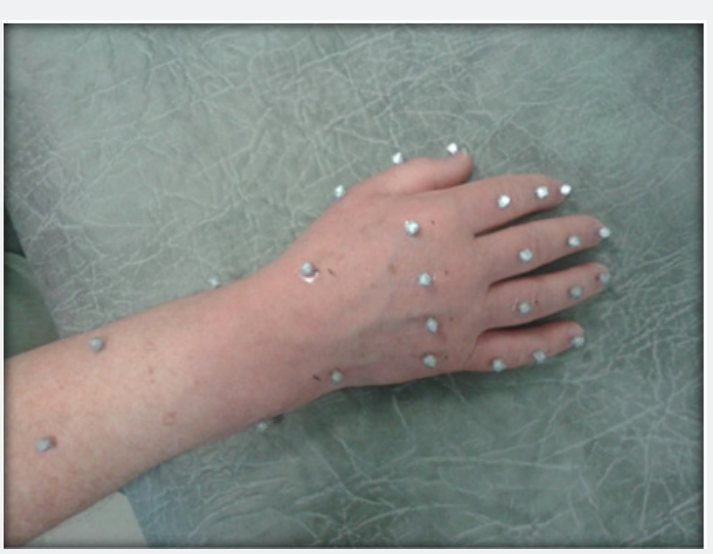

Figure 1: Placement of markers on the skin of the subjects in accordance with the Metcalf protocol.

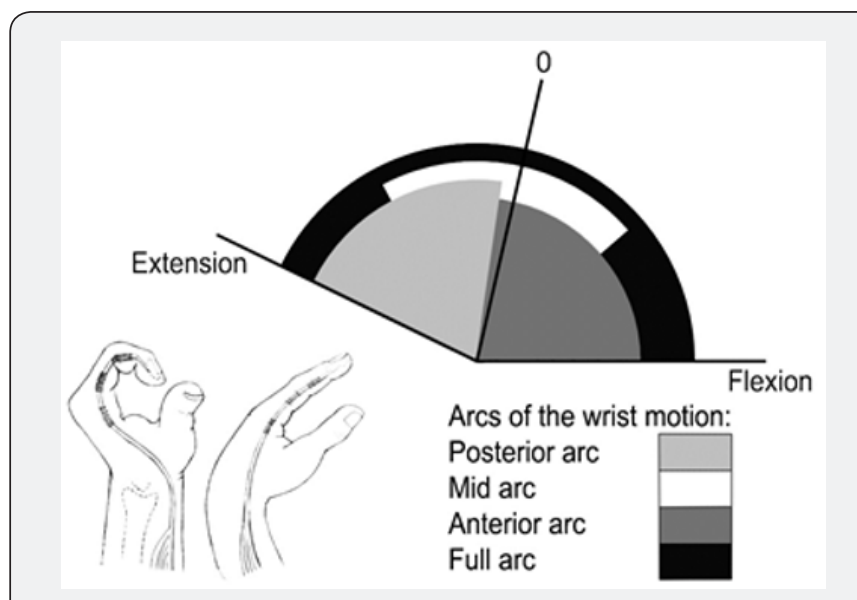

Figure 2: Division of wrist movements due to arcs. 


\section{Results}

During tenodesis phenomenon, recorded with the use of a three-dimensional motion analysis system BTS SMART, the mean motion of the wrist flexion (from a full extension to flexion) was $110^{\circ}\left(\mathrm{SD} \pm 12^{\circ}\right)$, whereas for the wrist extension motion (from a full flexion to a full extension) was $109^{\circ}\left(\mathrm{SD} \pm 12^{\circ}\right)$ on average (Table 1). The table (Table 1 ) also contains relative values enabling assessment of what percentage of TAM in a full motion constitutes TAM for arc. The results were also presented in Figure 3 (A-D) during the motion of both flexion and extension of the wrist for a better illustration of the differences. It turns out that the highest value of TAM for all fingers, both during flexion and extension of the wrist, occurs in the anterior arc, smaller or the same value in the mid arc and the smallest value always in the posterior arc.

Table 1: TAM indices values used for assessment of tenodesis exercises effectiveness and obtained for fingers II-V motion arcs.

\begin{tabular}{|c|c|c|c|c|c|c|c|c|c|c|c|c|c|c|c|c|}
\hline & \multicolumn{4}{|c|}{ Finger II } & \multicolumn{4}{|c|}{ Finger III } & \multicolumn{4}{|c|}{ Finger IV } & \multicolumn{4}{|c|}{ Finger V } \\
\hline & Full* & Ant..* & Mid* & Post.* & Full & Ant. & Mid & Post. & Full & Ant. & Mid & Post. & Full & Ant. & Mid & Post. \\
\hline Wrist flexion (o) & 110 & 67 & 55 & 43 & 119 & 70 & 61 & 49 & 128 & 79 & 61 & 48 & 118 & 72 & 61 & 47 \\
\hline Wrist flexion SD (o) & 23 & 15 & 16 & 17 & 21 & 17 & 16 & 19 & 22 & 16 & 15 & 16 & 27 & 18 & 19 & 22 \\
\hline Wrist extension (o) & 109 & 61 & 55 & 49 & 119 & 67 & 63 & 53 & 125 & 75 & 65 & 50 & 122 & 71 & 71 & 51 \\
\hline Wrist extension SD (o) & 24 & 17 & 18 & 12 & 21 & 19 & 16 & 12 & 25 & 22 & 16 & 10 & 31 & 27 & 20 & 11 \\
\hline Wrist flexion (\%) & 100 & 61 & 50 & 39 & 100 & 59 & 51 & 41 & 100 & 62 & 48 & 38 & 100 & 61 & 52 & 40 \\
\hline Wrist extension (\%) & 100 & 56 & 50 & 45 & 100 & 56 & 53 & 45 & 100 & 60 & 52 & 40 & 100 & 58 & 58 & 42 \\
\hline
\end{tabular}

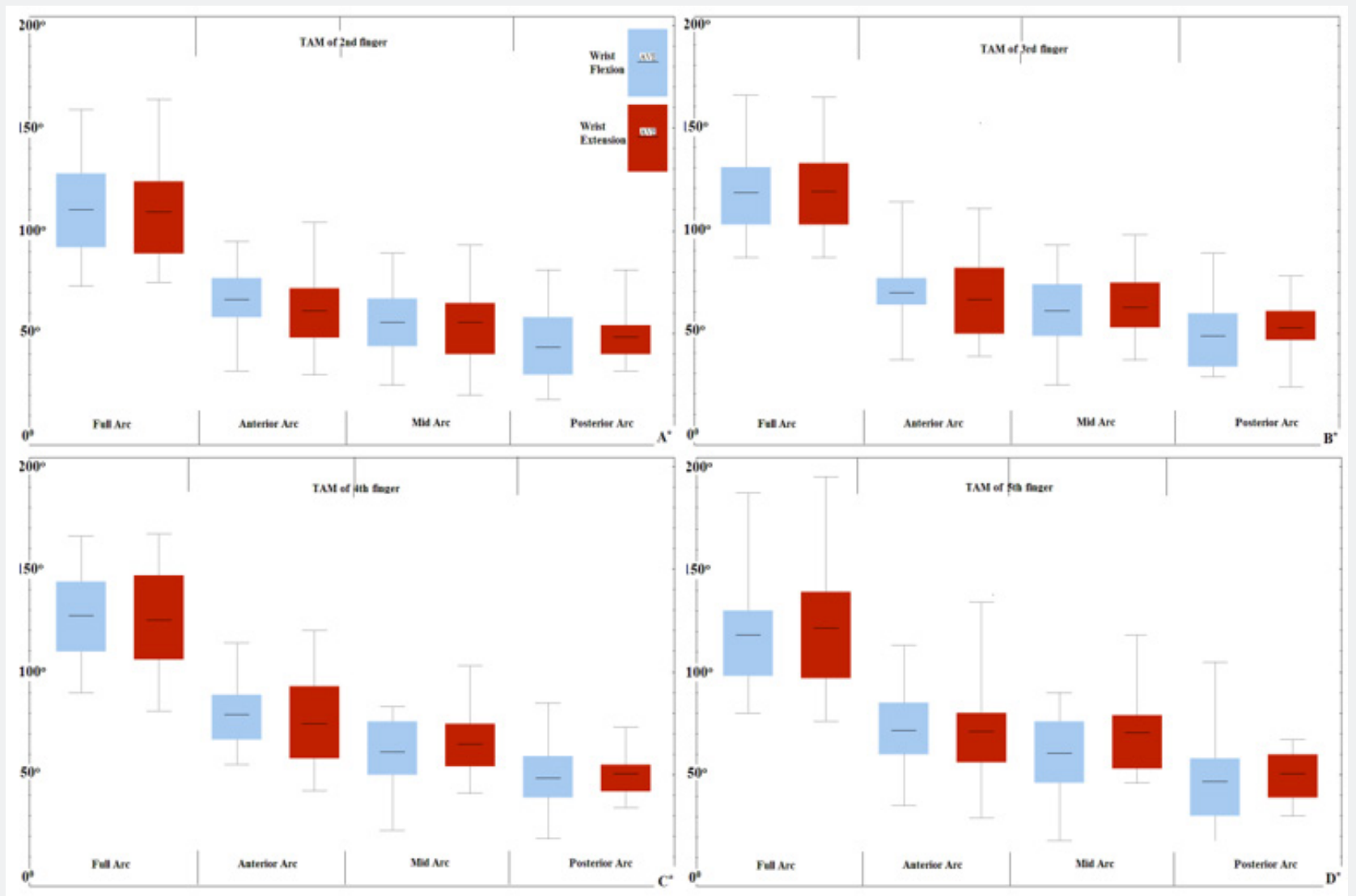

Figure 3: TAM of $2 n d\left(A^{*}\right), 3 r d\left(B^{*}\right), 4 \operatorname{th}\left(C^{*}\right)$ and $5 \operatorname{th}\left(D^{*}\right)$ fingers during flexion and extension of wrist in a full range of motion and anterior, mid and posterior arcs.

The results obtained for finger III in flexion and extension movements for anterior and mid arcs, as well as for finger IV during extension also for anterior and mid arcs are isolated exceptions. Motion arcs of the radiocarpal joint, also the TAM values obtained for flexion and extension movements were compared to find significant differences. In the anterior arc a statistically significant difference of TAM $(p<0.05)$ was found only for finger II. In the mid arc of motion there was observed 
a statistically significant difference of TAM $(\mathrm{p}<0.05)$ only for finger $\mathrm{V}$ (Table 2). The difference was $10^{\circ}$. However, it should be mentioned that for finger IV the result was close to the assumed level of statistical significance $(p=0.06)$. In this case the difference was $4^{\circ}$. The assessment of differences significance between TAM of fingers II-V during the tenodesis manoeuvre for extension and flexion movements was also conducted in a full range of the radiocarpal joint movement (Table 2). A statistically significant TAM difference $(\mathrm{p}<0.05)$ was found only for finger IV. The difference was $7^{\circ}$. A result close to the assumed significance level was observed for finger $\mathrm{V}$. The difference was $4^{\circ}$. Another phase of the analysis was to assess the TAM index differences between pairs of fingers in the wrist full arc.

Table 2: Differences of the TAM of $2^{\text {nd }}\left(A^{*}\right), 3^{\text {rd }}\left(B^{*}\right), 4^{\text {th }}\left(C^{*}\right)$ and $5^{\text {th }}\left(D^{*}\right)$ fingers during flexion and extension of wrist in a full range of motion and anterior, mid and posterior arcs.

\begin{tabular}{|c|c|c|c|c|c|c|c|c|c|}
\hline & \multirow{3}{*}{\multicolumn{2}{|c|}{ 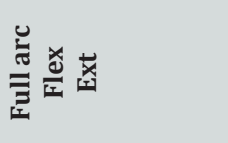 }} & \multicolumn{7}{|c|}{ Finger II } \\
\hline & & & \multicolumn{2}{|c|}{ Ant. arc } & \multicolumn{2}{|c|}{ Mid. arc } & \multicolumn{2}{|c|}{ Post. Arc } & \\
\hline & & & Flex & Ext & Flex & Ext & Flex & Ext & \\
\hline \multirow{8}{*}{ 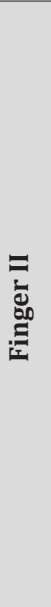 } & \multirow{2}{*}{ 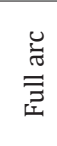 } & 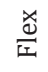 & & & & & & & \\
\hline & & 若 & 1 & & & & & & \\
\hline & \multirow{2}{*}{ 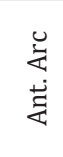 } & 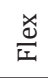 & $43^{*}$ & $43^{*}$ & & & & & \\
\hline & & 藏 & $49^{*}$ & $59 *$ & $6^{*}$ & & & & \\
\hline & \multirow{2}{*}{ 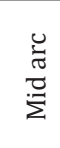 } & $\frac{\underset{0}{I}}{I}$ & $55^{*}$ & $54^{*}$ & $12^{*}$ & 6 & & & \\
\hline & & 若 & $55^{*}$ & $54^{*}$ & $11^{*}$ & 5 & 0 & & \\
\hline & \multirow{2}{*}{ 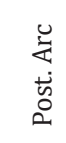 } & $\frac{\underset{0}{I}}{x^{\prime}}$ & $67^{*}$ & $55^{*}$ & $24^{*}$ & $18^{*}$ & $12^{*}$ & 12 & \\
\hline & & 芉 & $62^{*}$ & $61^{*}$ & $18^{*}$ & $12^{*}$ & 7 & 7 & 5 \\
\hline
\end{tabular}

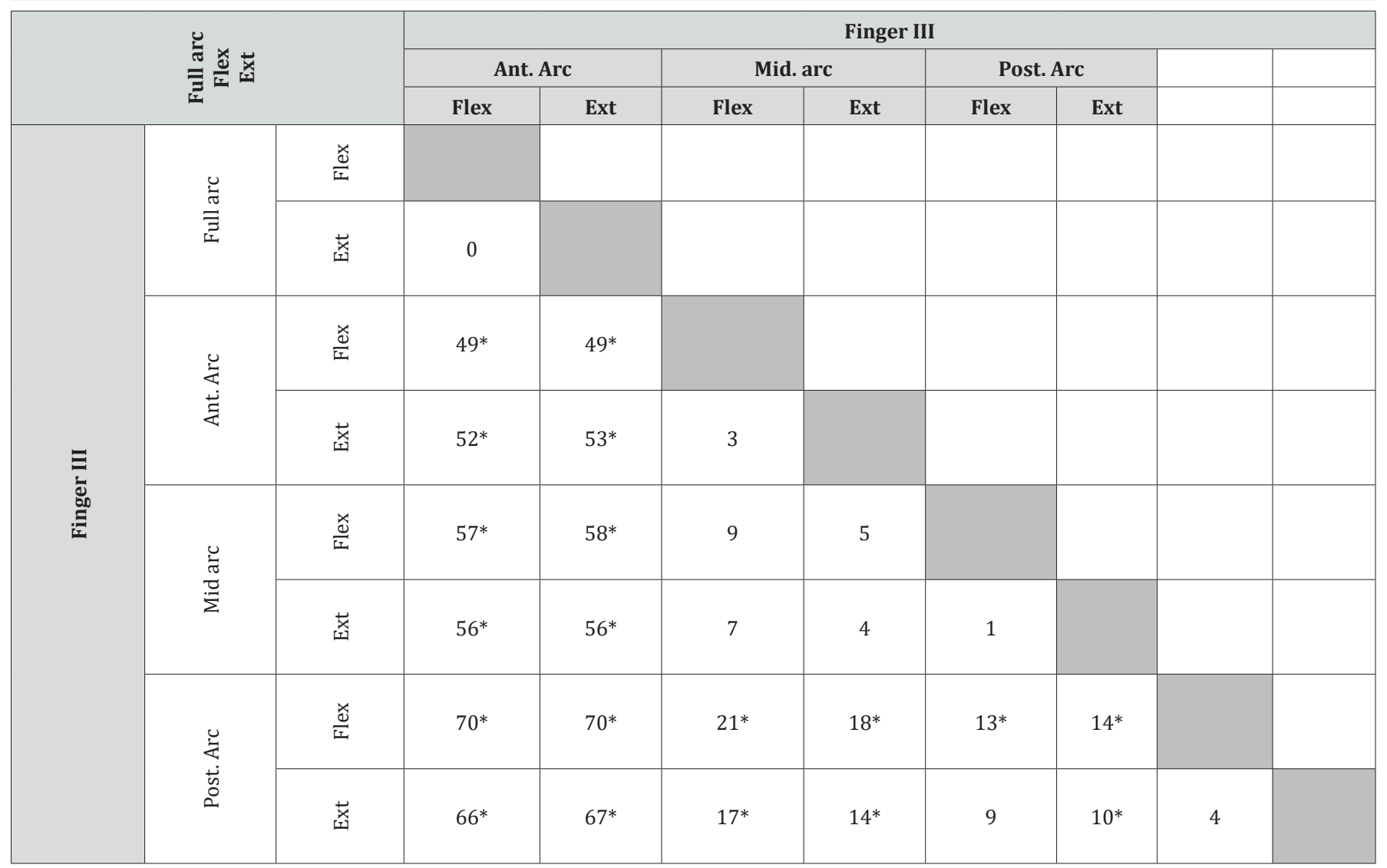


Orthopedics and Rheumatology Open Access Journal (OROAJ)

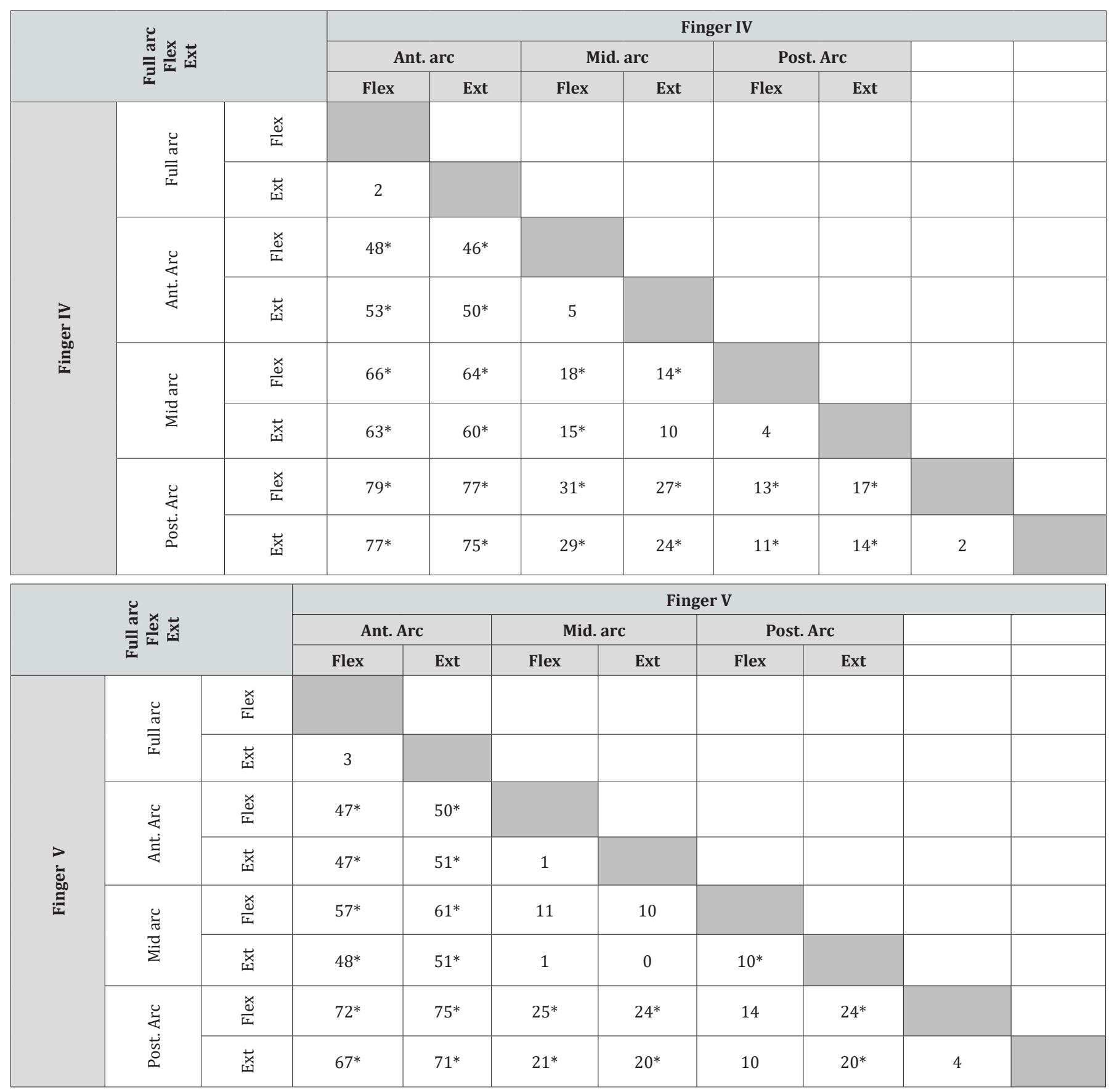

*- statistically significant results.

For a flexion movement, only differences of results for fingers III and IV were not statistically significant (Table 2). In the remaining combinations the differences of the compared measurements results were statistically significant. The highest difference was found between the TAM values of fingers II and III $\left(18^{\circ}\right)$, whereas for the other pairs with observed statistical significance the differences were close and amounted at $8^{\circ}$ or $9^{\circ}$. An analogical comparison of TAM differences for fingers pairs in extension motion presented only differences in results for combinations of finger II with other fingers (Table 2). The TAM values for fingers III, IV and V did not differ significantly.
Correlation values of mutual TAM indices for fingers in all motion arcs turned out to be high and statistically significant, both for flexion and extension movements (Table 3). However, no statistically significant correlations were found between the size of the motion angle in the wrist (extension and flexion) and the TAM values of fingers from II to $\mathrm{V}$ in full arc (Table 3). The tests also demonstrated that during voluntary, calm tenodesis movement performed by the examined in full arc, the duration of one exercise cycle was 2 seconds on average in each of the movement directions. 


\section{Orthopedics and Rheumatology Open Access Journal (OROAJ)}

Table 3: Correlation matrix between extension and flexion movements of the wrist and the TAM values of fingers II-V in extension and flexion. The results were obtained based on the BTS SMART device measurements.

\begin{tabular}{|c|c|c|c|c|c|c|c|c|}
\hline & TAM2Flx & TAM2Ext & TAM3Flx & TAM3Ext & TAM4Flx & TAM4Ext & TAM5Flx & TAM5Ext \\
\hline TAM2Flx & & & & & & & \\
\hline TAM2Ext & & & & & & & \\
\hline TAM3Flx & $0.77^{*}$ & & & & & & \\
\hline TAM3Ext & & $0.88^{*}$ & & & & & \\
\hline TAM4Flx & $0.76^{*}$ & & $0.85^{*}$ & & & & \\
\hline TAM4Ext & & $0.84^{*}$ & & $0.92^{*}$ & & & \\
\hline TAM5Flx & $0.77^{*}$ & & $0.75^{*}$ & & $0.84^{*}$ & & \\
\hline TAM5Ext & & $0.80^{*}$ & & $0.86^{*}$ & & $0.88^{*}$ & \\
\hline WristFlx & 0.16 & & -0.04 & & -0.04 & & 0.08 & \\
\hline WristExt & & 0.36 & & 0.19 & & 0.18 & \\
\hline
\end{tabular}

\section{Discussion}

Excursion of flexor tendons has often been assessed in professional literature on corpses Cooney et al. [17]. Ugbolue et al. [18]. Yamaguchi et al. [19], Kociolek \& Keir [20] and on living humans, mainly with the use of ultrasonic techniques Wehbé \& Hunter [21,22], Lopes et al. [23], Kursa et al. [24], Martin et al. [25], Oh et al. [26], Yoshii et al. [27]. Ubgoule et al. [18] assessed, among others, changes occurring in FDP and FDS tendons during movements performed in MCP joints. Lopes et al. investigated reactions of FDS flexors tendons during complex movements of the fingers and wrist. Armstrong and Chaffin (after Kociolek \& Keir [20], and Lopes et al. [23] created a model predicting excursions of FDP and FDS flexor tendons relying, among others, on angular changes in joints of corpses. However, there are no studies estimating performance of fingers and joints during movements of the wrist with relaxed fingers i.e. during, so called, tenodesis movements.

Tenodesis exercises are introduced at the early phase of restitution after tendons anastomosis. Formby 7 recommends application of tenodesis exercises after injury of fingers tendons in zones I $-\mathrm{V}$ with the use of a special apparatus (Hinged splint). The protocol suggests limiting the wrist extension to 30 degrees and a possibility of its maximal flexion. MCP joints remain within 60 degrees of flexion, whereas IP joints can be maximally extended. Similarly, in "The Indiana Protocol" the values of 30 degrees for the wrist extension, its maximal flexion and maintaining MCP joints within 50 degrees of flexion [10] are given as border values. Many other protocols or authors mention the necessity to apply tenodesis exercises discussing flexion and extension movements of the wrist, but they do not specify any border values $[11,28]$. There can appear many modifications of tenodesis exercises relying on, e.g. combining them with "placeand-hold" exercises or increasing MCP joints extension [11,12].

The appearance of values of motion ranges in the wrist during tenodesis phenomenon lower than during goniometric measurements, which was observed in the tests, resulted from the fact that in case of goniometric measurement the movements were performed in forced conditions in a full range of mobility (according to the standards presented by the SFTR method), whereas tenodesis movements were performed naturally, without forcing the conditions.

The obtained results of the tests indicated changes occurring in fingers joints that for an easier analysis were illustrated by the TAM values (Total Active Motion), following the formula suggested by the American Society for Surgery of the Hand [9]. The highest TAM values of fingers II - V were observed during performing movements of the wrist in a full arc that amounted at 154 degrees on average. The result complied with former expectations and the obtained TAM values ranged between 109 and 128 degrees, which constitutes, respectively, 42 and $49 \%$ of the full TAM of fingers [29-31].

Despite considerable changes of motion ranges in fingers joints, the statistically significant differences between flexion and extension movements were found only in finger IV. During flexion of the wrist, higher angle changes also took place. Whereas, a slightly higher TAM was observed in finger $\mathrm{V}$ during extension movements of the wrist. The values obtained in fingers II and III were practically identical, regardless of what movement of the wrist was performed. Yet, it should be emphasized that in therapeutic practice the motion range of fingers is often increased during tenodesis exercises by their passive flexion during extending the wrist and PIP and DIP joints extension during flexing it5,6.

However, if we look at changes of the range of motion of fingers while performing movements of the wrist in smaller arcs, the slightest changes of TAM occur in the posterior arc. Such a result means that from the perspective of the TAM index, the exercises performed in the posterior arc are the least effective. A value of this observation is particularly important for clinical practice as it is the posterior arc of motion where the highest loads of fingers flexors tendons resulting from their extension can be expected bringing some risks for patients. A slightly higher TAM range is found during the extension movement ( 49 - 53 degrees), but the existing differences (between the wrist 
flexion and extension) do not indicate statistical significance. For this arc of the wrist movement the changes of motion ranges of fingers (TAM) were close to each other.

Highest changes of fingers TAM were registered during performing movements of the wrist in the mid arc. (flexion of the wrist 69 - 43, extension 69 - 43). Proceeding from finger II to $\mathrm{V}$ there is a progressively increasing TAM difference of fingers during the wrist extension movement (subsequently: 55, 63, 65 and 71 degrees). The difference in the range of motion of fingers between values during flexing and extending the wrist was statistically significant only for finger $\mathrm{V}$, and in case of finger IV it was at the border of the assumed significance $(p=0.06)$. During extending the wrist, the obtained ranges of fingers motion were close and amounted at 55 degrees for finger II and 61 degrees for the remaining ones. If we put together the obtained values and the TAM values of fingers in a full motion arc of the wrist, it turns out that proceeding from finger II to $\mathrm{V}$ we obtain higher and higher values. In case of finger II it is 55\% for flexion and for extension in comparison to a full arc of the wrist motion. For finger III the values are, respectively, 51 and 53\%, for IV 48 and $54 \%$, and for V 52 and 58\%.

The highest changes in the motion range of fingers II-V were observed during tenodesis movements in anterior arc. Here, in turn, higher values were found during the wrist flexion than during its extension to a neutral position. Differences between flexion and extension were statistically significant only in finger IV. However, this finger featured the least changes of the TAM range in comparison to the other fingers. The highest changes occurred in finger IV - 79 degrees in flexion and 75 in extension. An analysis of the tests results also demonstrated existence of "different effectiveness" illustrated by the TAM value in different arcs of the wrist movement. The results may suggest that tenodesis exercises should not be applied in a high range of motion as it does not cause a remarkable increase of fingers TAM, whereas it may lead to a growth of anastomosed structures tensions.

A lack of correlation of TAM and the range size in which the movement in the wrist takes place that was found in the examined group may suggest that the exercise effectiveness measured by the TAM index is not affected by the range of movement performed in the wrist. The result might be surprising as it could be expected that an increase of the range in which movement takes place in the wrist will be accompanied by growing values of movement ranges in fingers (TAM). Therefore, it can be stated that an increase of the motion range does not cause statistically significant growth of the exercise effectiveness. In the future it would be advisable to estimate how TAM for fingers alters with a change of the motion range in the wrist. It is likely to grow to some values, and next it does not change significantly, so further increase of the motion range in the wrist is then pointless. The relation of TAM and the angle value in the wrist is probable of a non-linear character. However, such relation was not examined in the work. It would be also possible to divide a full range of motion available in each patient into smaller sections (e.g. 10\% of the full range) and check what changes of TAM occur in each of the smaller parts.

Based on the conducted tests it can be concluded that tenodesis exercises in a given patient will present similar effectiveness for all fingers measured by the TAM values. The tests results are based on recording measurements in healthy people. Thus, it is difficult to draw conclusions concerning the character of the analysed values in a group of patients with tendons injuries. A more thorough analysis of the phenomenon requires further clinical trials.

\section{Conclusion}

i. No statistically significant correlation was found between the TAM indices describing angular changes occurring in joints of fingers II-V and the motion range in the wrist. It suggests that increasing the motion range in the wrist above certain values does not affect effectiveness of exercises.

ii. Different effectiveness of exercises was observed in different motion arcs in the wrist. The highest effectiveness was found in the anterior arc, and the least one in the posterior arc. Such a result suggests that it is recommended to limit the range of performed tenodesis exercises in the posterior arc as in this arc the highest load of fingers flexors tendons can be expected.

iii. Strong correlations of the TAM index between fingers were observed which shows that in a given patient the level of effectiveness of performed tenodesis exercises is similar for individual fingers.

iv. It is advisable to perform studies over the phenomenon of tenodesis pertaining to the size of tendons tension with the use of advanced mathematical models including muscles. Such tests could lead to defining optimal, regarding effectiveness of tenodesis, exercises and at the same time safe range of the wrist motion.

\section{References}

1. Bujega M, Mifsud M, Zammit J (2016) Functional results following surgical repair of post-traumatic hand tendon injuries. Malta Medical Journal 28(2).

2. Googins T, Williams S, Rehabilitation of the hand. Orthopaedics and Trauma 33(1): 62-65.

3. Liechti DJ, Mitchell JJ, Menge TJ, Hackett TR (2018) Immediate physical therapy without postoperative restrictions following open subpectoral biceps tenodesis: low failure rates and improved outcomes at a minimum 2-year follow-up, J Shoulder Elbow Surg 27(10): 1891-1897.

4. Newton A, Tonge X, Hawkes D, Bhalaik V (2019) Key aspects of anatomy, surgical approaches and clinical examination of the hand, Orthopaedics and Trauma 33(1): 1-13. 
5. Alonso-Rasgado T, Zhang Q Jimenez-Cruz D, Bailey C, Pinder E, et al (2018) Evaluation of the performance of three tenodesis techniques for the treatment of scapholunate instability: flexion-extension and radial-ulnar deviation. Med Biol Eng Comput 56(6): 1091-1105.

6. Thoreson AR, Rappaport PO, Yang TH, Reisdorf RL, Zhao C (2019) Calculation of flexor pollicis longus moment arm for wrist motion in a cadaver model validates the tenodesis effect for therapy. J Hand Ther pii: S0894-1130(18)30209-36.

7. Formby M (2006) Flexor Tendon Repair" w Burke SL, Higgins JP, McClinton MA, Saunders RJ, Valdata L "Hand and Upper Extremity Rehabilitation. A practical guide; ( $3^{\text {rd }}$ edn)” Elsevier 227-244.

8. Gail N Growth (2005) Current practice patterns of flexor tendon rehabilitation. J Hand Ther 8(2): 169-174.

9. Libberecht K, Lafaire C, Van Hee R (2006) Evaluation and Functional Assessment of Flexor Tendon Repair in the Hand. Acta Chirurgica Belgica 106(5): 560-565.

10. Vucekovich K, Gallardo G, Fiala K (2005) Rehabilitation after flexor tendon repair. Reconstruction and tenolysis. Hand Clinics 21(2): 257 265.

11. Department of Rehabilitation Services. The Brigham and Women's Hospital.

12. Evans RB (2012) Managing the Injured Tendon: Current Concepts. J Hand Ther 25(2): 173-190.

13. Gelberman RH, Woo SL, Lothringer K, Akeson WH, Amiel D, et al (1982) Effects of early intermittent passive mobilization on healing canine flexor tendons. Journal of Hand Surgery (American Volume) $7(2): 170-175$.

14. Clancy SP, Mass DP (2013) Current flexor and extensor tendon motion regimens: a summary, Hand Clinics 29(2): 295-309.

15. Starr HM, Snoddy M, Hammond KE, Seiler JG (2013) $3^{\text {rd }}$ Flexor tendon repair rehabilitation protocols: a systematic review. J Hand Surg Am 38(9): 1712-1717.

16. Metcalf CD, Notley SV, Chappell PH, Burridge JH, Yule VT (2008) Validation and application of a computational model for wrist and hand movements using surface markers. Biomedical Engineering 55(3): 1199-1210.

17. Cooney WP, Lin GT, An KN (1989) Improved Tendon Excursion Following Flexor Tendon Repair. Journal of Hand Therapy 2(2): 102106

18. Ugbolue UC, Hsu WH, Goitz RJ, Li ZM (2005) Tendon and nerve displacement at the wrist during finger movements. Clinical Biomechanics 20(1): 50-56.
19. Yamaguchi T, Osamura N, Zhao C, An KN, Amadio PC (2008) Relative Longitudinal Motion of the Finger Flexors. Subsynovial Connective Tissue and Median Nerve before and after Carpal Tunnel Release in a Human Cadaver Model. Journal of Hand Surgery (American Volume) 33(6): 888-892.

20. Kociolek AM, Keir PJ (2011) Modelling tendon excursions and moment arms of the finger flexors: Anatomic fidelity versus function. Journal of Biomechanics 44: 1967-1973.

21. Wehbé MA, Hunter JM (1985) Flexor tendon gliding in the hand. Part I. In vivo excursions. Journal of Hand Surgery 10(4): 570-574.

22. Wehbé MA, Hunter JM (1985) Flexor tendon gliding in the hand. Part II. Differential gliding. Journal of Hand Surgery 10(4): 575-579.

23. Lopes MM, Lawson W, Scott T, Keir PJ (2011) Tendon and nerve excursion in the carpal tunnel in healthy and CTD wrists. Clinical Biomechanics 26(9): 930-936.

24. Kursa K, Lattanza L, Diao E, Rempel D (2006) In Vivo Flexor Tendon Forces Increase with Finger and Wrist Flexion during Active Finger Flexion and Extension. J Orthop Res 24(4): 763-769.

25. Martin JR, Paclet F, Latash ML, Zatsiorsky VM (2013) Changes in the Flexor Digitorum Profundus Tendon Geometry in the Carpal Tunnel Due to Force Production and Posture of Metacarpophalangeal Joint of the Index Finger: An MRI Study. Clin Biomech (Bristol, Avon) 28(2): 157-163.

26. Oh S, Belohlavek M, Zhao C, Osamura N, Zobitz ME (2007) Detection of Differential Gliding Characteristics of the Flexor Digitorum Superficialis Tendon and Subsynovial Connective Tissue Using Color Doppler Sonographic Imaging. Journal of Ultrasound in Medicine 26(2): 149-155.

27. Yoshii Y, Villarraga HR, Henderson J, Zhao C, An KN (2009) Speckle Tracking Ultrasound for Assessment of the Relative Motion of Flexor Tendon and Subsynovial Connective Tissue in the Human Carpal Tunnel. Ultrasound in Medicine and Biology 35(12): 1973-1981.

28. Sandow M, Kay S (2003) Flexor tendon injuries w Prosser R. Conolly W.B. Rehabilitation of the Hand and Upper Limb. Elsevier 46-52.

29. Gelberman RH, Siegel DB, Woo SL, Amiel D, Takai S, et al. (1991) Bone Healing of digital flexor tendons: importance of the interval from injury to repair. A biomechanical, biochemical and morphological study in dogs J Bone Joint Surg Am 73(1): 66-75.

30. May EJ, Silfverskiöld KL, Sollerman CJ (1992) Controlled mobilization after flexor tendon repair in zone II: a prospective comparison of three methods. J Hand Surg Am17(5): 942-952.

31. Norkin CC, White DJ (2009) Measurement of Joint Motion. A Guide to Goniometry. ( $4^{\text {th }}$ Edn) FA Davis Company, Philadelphia, USA.

\section{Your next submission with Juniper Publishers will reach you the below assets}

- Quality Editorial service

- Swift Peer Review

- Reprints availability

- E-prints Service

- Manuscript Podcast for convenient understanding

- Global attainment for your research

- Manuscript accessibility in different formats

( Pdf, E-pub, Full Text, Audio)

- Unceasing customer service

Track the below URL for one-step submission https://juniperpublishers.com/online-submission.php 